This is an Open Access article distributed under the terms of the Creative Commons Attribution-Noncommercial License, which permits unrestricted use, distribution, and reproduction in any noncommercial medium, provided the original work is properly cited.

\title{
SPICA'S CHALLENGE: PROBING HIGH-REDSHIFT UNIVERSE TOWARD THE EPOCH OF REIONIZATION
}

\author{
E. Egami \\ Steward Observatory, University of Arizona, 933 N. Cherry Ave., Tucson, AZ 85721, USA
}

\begin{abstract}
In the coming years, we expect to see ALMA and JWST pushing the frontier of extragalactic IR/submm astronomy beyond the current limit of $z \sim 4$ and probably up to $z \sim 10$, the epoch of cosmic reionization. The main challenge for SPICA will be whether it can also probe such a high-redshift regime. Here, I discuss such a possibility with various observing strategies, showing that SPICA has a great potential to explore the $4<z<10$ Universe.
\end{abstract}

Key words: Galaxies: high-redshift - Infrared: galaxies

\section{INTRODUCTION}

Observational cosmology is now on the verge of making another breakthrough in the history of science: to identify the first generation of galaxies that appeared and reionized the intergalactic space, thereby ending the so-called "Dark Ages" of the Universe. This process, called "cosmic reionization", marks a major phase transition of the Universe, after which the intergalactic space has become transparent to $\mathrm{HI}$ ionizing radiation. Recent WMAP observations indicate that cosmic reionization took place around $\mathrm{z} \sim 10$ (e.g., Dunkley et al., 2009) while QSO absorption line studies suggest that the reionization process is not complete to $z \sim 6$ (e.g., Fan et al., 2006). Our challenge, therefore, is to understand how galaxies formed and evolved during this critical epoch in the history of the Universe.

Deep optical and near-/mid-infrared observations are now routinely detecting galaxies up to a redshift of 7 and even beyond, providing a first glimpse of galaxy populations near the epoch of reionization. Preliminary results suggest that the rest-frame UV luminosity density (and therefore the UV-derived star formation rate density (SFRD)) is sharply declining from $z \sim 2-3$ to $z \sim 8$ (Figure 1). The next question is whether this observed sharp decline reflects the true history of cosmic star formation or it is an artifact produced by dust extinction. For example, when $z \sim 3-4$ Lyman Break Galaxies (LBGs) were first studied, a similar decline was seen at $z \sim 3-4$, but it was later understood as the effect of dust extinction. In fact, when corrected for dust extinction, the UV-derived SFRD became much flatter at $z \sim 1-4$ (e.g., Steidel et al., 1999).
Furthermore, even heavier dust extinction could make galaxies drop out of optical (i.e., rest-frame UV) surveys all together (e.g., LBG surveys), and indeed such a population of luminous but heavily dust-obscured galaxies have been discovered by submillimeter observations (e.g., Smail et al., 2002). This leads to a question we hope to answer with SPICA: "Did dust-obscured (i.e., IR-luminous) galaxies play an important role in the early history of the Universe at $4<z<10$ ?" The lower bound of $z \sim 4$ corresponds to the current limit of IR-luminous galaxy searches; The upper bound of $z \sim 10$ marks the epoch of reionization. A population of dust-obscured galaxies could certainly hide a substantial amount of star-forming activities at $z>4$ from optical/near-IR observations, although its relevance to cosmic reionization may be a separate question since dust-obscured galaxies would probably not release intergalactic $\mathrm{HI}$ ionizing radiation efficiently.

The abundance and properties of dust-obscured galaxies at $4<z<10$ are virtually unknown. The SPICA's goal, therefore, is to identify such a population of galaxies and characterize the physical properties of individual detected sources.

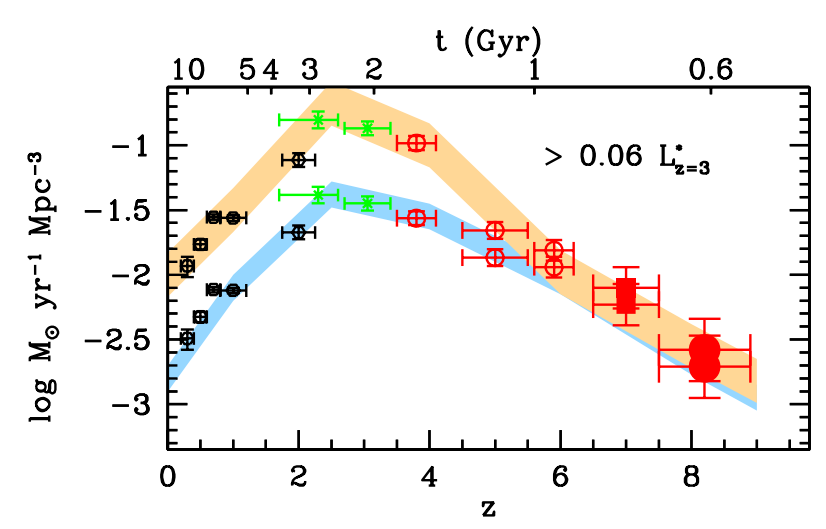

Figure 1. Determinations of the $U V$ luminosity density and SFR density - integrated to $0.06 L_{z=3}^{*}(-18$ AB mag) - as a function of redshift. The lower set of points (and blue region) show the SFR density determination inferred directly from the $U V$ light, and the upper set of points (and orange region) show what one would infer using dust corrections inferred from the $U V$-continuum slope measurements (Bouwens et al., 2009). Note, however, that such UV-based dust corrections will not reveal a luminous but heavily dust-obscured stellar population. 


\section{Beyond HERSChEL AND JWST}

Over the next several years, Herschel will produce many exciting results through far-IR/submm observations. After that, JWST will start conducting near-/mid-IR observations to unprecedented depths. SPICA, therefore, must offer powerful observing capabilities that will exceed those of Herschel and JWST.

Beyond Herschel — Penetrating through the confusion limit with spectroscopic observations: Despite its greatly improved sensitivities, it will be difficult for Herschel to probe the Universe at $z>2-3$ because of its confusion limits. For example, a ULIRG with an infrared luminosity of $5 \times 10^{12} \mathrm{~L} \odot$ will fall below the expected Herschel confusion limits when they are at $z>2-3$.

Note that having a similar telescope size, SPICA is also bounded by the same limits. In other words, as far as broad-band imaging is concerned, little gain is expected with SPICA at $>100 \mu \mathrm{m}$, where Herschel images are already confusion-limited. SPICA's cold telescope alone will not necessarily allow us to probe the high-redshift Universe deeper than with Herschel.

SPICA's strength, therefore, must be its excellent spectroscopic capabilities, whether it is FTS spectro-imaging with SAFARI or grating spectroscopy with BLISS. To go beyond Herschel and achieve the ultimate backgroundlimited sensitivities offered by SPICA's cold telescope, it is necessary to exploit spectroscopic information (e.g., detection of strong PAH features and fine-structure/molecular hydrogen lines), which will enable us to detect sources deeper into the confusion (e.g., Clements et al., 2007).

Beyond JWST - Surveying a wider areas with faster mapping: Compared with JWST, SPICA has a smaller telescope but its thermal background is much lower. As a result, the sensitivities of MIRACLE and MIRI are comparable at $5-28 \mu \mathrm{m}$, where both instruments operate. MIRI is expected to surpass MIRACLE at $<20 \mu \mathrm{m}$ slightly while the reverse will be the case at $>20 \mu \mathrm{m}$.

When it comes to covering a wider area, however, MIRACLE is much more effective because its field of view (FOV) is so much larger $\left(6^{\prime} \times 6^{\prime}\right)$ than that of MIRI $\left(1.7^{\prime} \times 1.3^{\prime}\right)$. This difference $(\times 16)$ is more than enough to compensate for the difference in the collecting areas $(\times \sim 4)$.

Another advantage MIRACLE may have over MIRI is a suite of narrow-band filters and slit masks that are currently planned. For example, MIRACLE will be powerful for searching high-redshift line emitter (e.g., $z>7 \mathrm{H} \alpha$ emitters).

Finally, it should be noted that the $30-70 \mu \mathrm{m}$ range is where neither Herschel nor JWST will cover.

\section{ExAmples of SPICA High-Redshift OBservations}

As stated above, SPICA's goal will be twofold: (1) to identify $4<z<10$ IR-luminous galaxies, and (2) to characterize the physical properties of individual detected sources.
Below, we list some examples of such SPICA observations. For (2), we expect a number of interesting targets to be known beforehand from various ALMA/JWST deep surveys as well as from the SPICA surveys mentioned below.

\subsection{IDENTIFY $4<z<10$ IR-LUMINOUS GALAXIES}

SAFARI Spectro-imaging survey of $7.7 \mu \mathrm{m}$ PAH emitters: The $7.7 \mu \mathrm{m}$ PAH complex is the most luminous and conspicuous emission feature seen in the rest-frame mid-IR spectra of star-forming galaxies. For example, the $7.7 \mu \mathrm{m}$ PAH features of $z \sim 2$ star-forming submillimeter galaxies (SMGs) often have luminosities above $\sim 10^{10} \mathrm{~L}_{\odot}$ (Figure 2). Note that the PAH feature is broad, requiring only a low resolving power $(\mathrm{R} \sim 20)$ for its detection. With a flux sensitivity of $10^{-19} \mathrm{Wm}^{-2}$ in the low-resolution FTS mode, SAFARI can conduct an efficient survey of such strong $7.7 \mu \mathrm{m}$ PAH emitters up to $z \sim 10$.

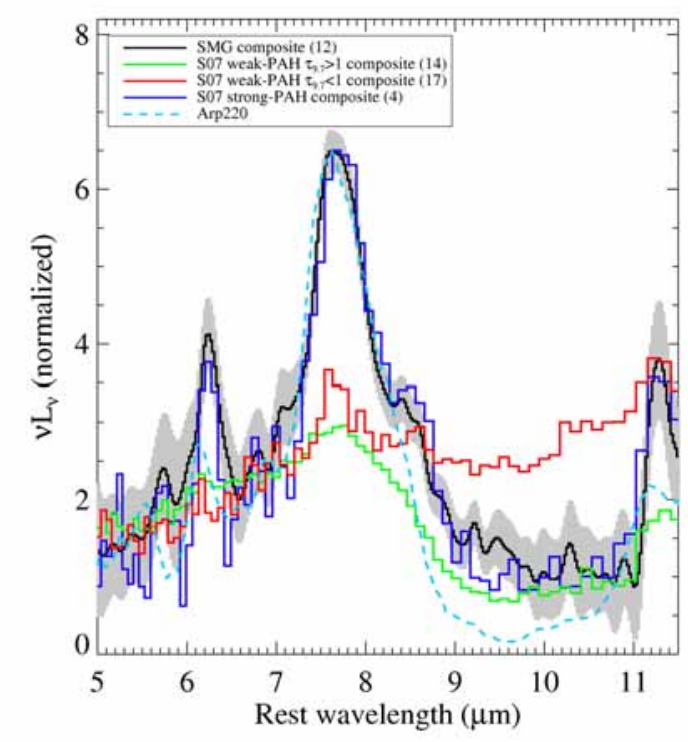

Figure 2. Strong 7.7 $\mu \mathrm{m}$ PAH feature detected in $z \sim 2$ SMGs (Pope et al., 2008).

A potential problem for such a $\mathrm{PAH}$ emitter survey will be a systematic decrease of metallicity at high redshift. Spitzer observations have shown that the PAH feature strength will decrease significantly for low metallicity galaxies (Engelbracht et al., 2008), and this effect could reduce the effectiveness of a PAH emitter survey at high redshift, where we might expect systematically lower metallicity environments. On the other hand, such a PAH emitter survey will detect only the most luminous galaxies at high redshift, so this metallicity effect may be less significant if the change of the mass-metallicity relation is less dramatic with luminous massive galaxies (Maiolino et al., 2008). Furthermore, known $z \sim 6$ QSOs show no sign of a decreased metallicity. Therefore, we have some reason to 
suspect that a PAH emitter survey will successfully find high-redshift galaxies although a null detection would also be quite interesting scientifically.

MIRACLE Narrow-band imaging/multi-slit survey of $\mathbf{H} \alpha$ emitters: $\mathrm{H} \alpha$ is the brightest hydrogen recombination line in the rest-frame optical, and therefore will serve as a good marker for high-redshift galaxies. $\mathrm{H} \alpha$ will be redshifted into the MIRACLE passband at $z \sim 7(5.25 \mu \mathrm{m})$. With specifically designed narrow-band filters/slitmaks together with the large FOV, MIRACLE can conduct an efficient survey of $\mathrm{H} \alpha$ emitters at $z>7$. Note that such a $\mathrm{H} \alpha$ survey will detect not only dusty IRluminous galaxies but also UV-bright galaxies with minimal dust extinction and strong line emitters such as giant blobs found with Ly $\alpha$ emission.

\subsection{Characterize individual Detected sources}

BLISS Fine-structure $/ \mathbf{H}_{2}$ line spectroscopy: Some low-redshift luminous galaxies (e.g., $z \sim 0.2$ brightest cluster galaxies in strongly cooling cluster cores) show very strong fine-structure (e.g., [Ne II] $12.8 \mu \mathrm{m}$ ) and $\mathrm{H}_{2}$ lines with luminosities above $\sim 10^{9} L_{\odot}$ (Figure 3 ). With a flux sensitivity of $10^{-20} \mathrm{Wm}^{-2}$ in the medium-resolution ( $\mathrm{R} \sim 700)$ mode, BLISS can detect such strong lines up to $z \sim 10$, providing information on the physical properties of ionized gas components as well as those of $\mathrm{H}_{2}$-emitting regions. Although it is by no means clear that similarly luminous line-emitting galaxies would exist at such high redshift, if cluster cooling flows are triggering these luminous lines, a similar physical process might be at work in high-redshift forming galaxies.

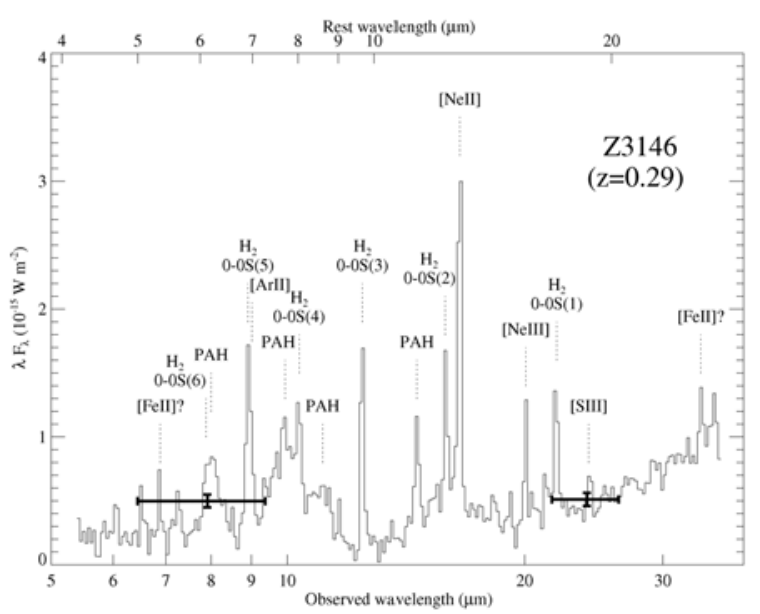

Figure 3. Strong [Ne II] fine-structure line as well as abnormally luminous $\mathrm{H}_{2}$ lines detected in the brightest cluster galaxy in Zwicky 3146, an X-ray-luminous galaxy cluster in which the existence of a cluster cooling flow is suspected (Egami et al., 2006).
MIRACLE rest-frame optical spectroscopy: MIRACLE can perform rest-frame optical spectroscopy of highredshift galaxies to diagnose their properties by using various line ratios, although JWST has a similar capability.

\section{SPICA Lensing Survey}

Although the examples above clearly demonstrate the potential power of SPICA to probe high-redshift Universe, it should be remembered that for galaxies to be detected by SPICA at $z>4$, they must be quite luminous intrinsically, such as cluster BCGs at $z \sim 0.2$ and SMGs at $z \sim 2$. However, under the hierarchical structure formation theories now widely adopted (e.g., $\Lambda \mathrm{CDM}$ ), we might expect such luminous systems to be less abundant at high redshift. In fact, such a scenario is consistent with the dimming of the bright end of the UV luminosity function currently observed at $z>3$ (Bouwens et al., 2009). If this is really the case, it will make it difficult for SPICA to detect $z>4$ galaxies in blank-field surveys.

One possible solution may be to conduct a lensing survey targeting massive clusters of galaxies. By observing the fields of massive lensing clusters and thereby taking advantage of their great gravitational lensing power, we can increase the sensitivities by up to a factor of 20-30. Note that both SAFARI and MIRACLE have FOVs that are large enough to contain the strongly lensed region in the cluster core (radius $\sim 1^{\prime}$ ).

The strong gravitational lensing of the distant Universe by intervening galaxy clusters has been exploited at many wavelengths to push the luminosity limits and redshifts out to which one can study evolving galaxies. In the $\mathrm{IR} /$ submm, lensing clusters act as transparent lenses, and cluster surveys at these wavelengths have been particularly powerful at, (a) digging below the confusion limits of submm surveys to constrain the faint end of the source counts, and (b) opening up the study of not only some of the most distant objects, but also those that are less extreme at slightly modest redshifts.

In fact, we are currently undertaking such a lensing survey using Herschel ("Herschel Lensing Survey"; PI: Egami). Simulated PACS $100 \mu \mathrm{m}$ and SPIRE $250 \mu \mathrm{m}$ images are shown in Figure 4, illustrating the remarkable power of gravitational lensing in the cluster core, pulling out a number of $z>2$ galaxies from the confusion noise.

With SPICA, we can conduct a similar survey using the FTS spectro-imaging mode of SAFARI. When the excellent line flux sensitivity of SAFARI $\left(\sim 10^{-19} \mathrm{~W} \mathrm{~m}^{-2}\right)$ is combined with the strong lensing power of massive galaxy clusters, we will be able to probe the existence/properties of IR-luminous galaxies in the epoch of cosmic reionization for the first time in the mid-/far-infrared.

More quantitatively, if we assume that the infrared galaxy luminosity function is unchanged at $z \sim 2$ and beyond, we may expect to detect $\sim 23$ infrared-luminous galaxies at $7<z<10$ via the $7.7 \mu \mathrm{m} \mathrm{PAH} \mathrm{feature} \mathrm{with}$ 

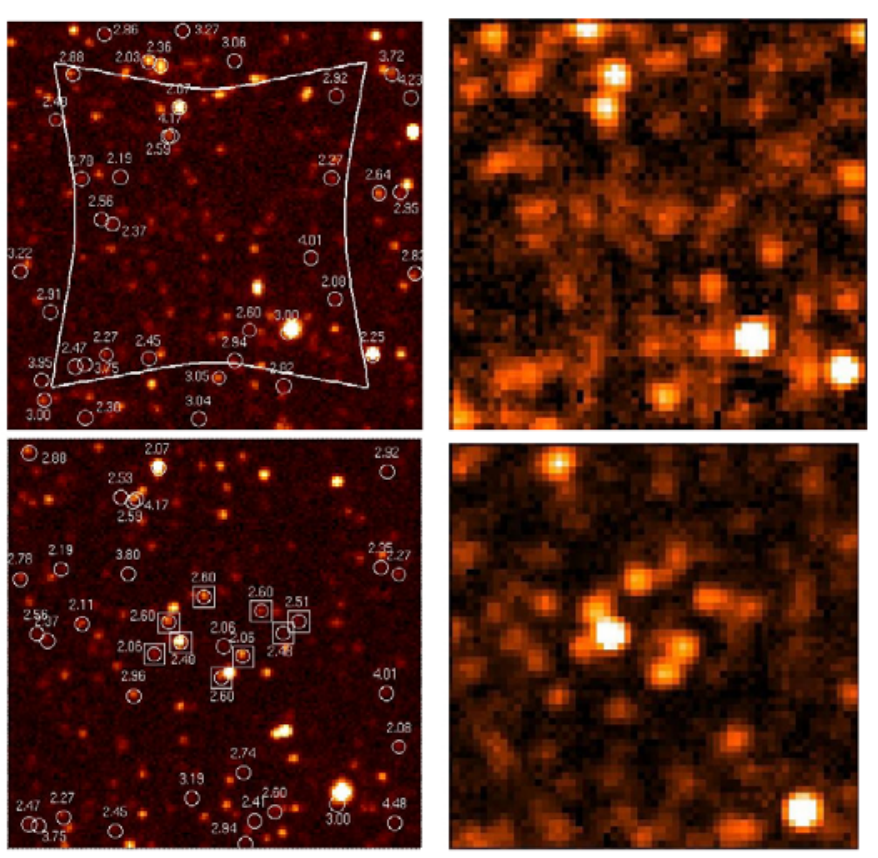

Figure 4. Simulated PACS $100 \mu \mathrm{m}$ images (left column) and SPIRE $250 \mu \mathrm{m}$ images (right column). The upper panels show the images of a blank field (' ' $\times 7^{\prime}$ ') while the lower panels show the same field gravitationally lensed by the typical lensing potential of a massive galaxy cluster. The numbers shown in the PACS images are the redshifts of the sources, and the small squares denote those sources that are amplified by more than a factor of 5. The distorted square shown in the upper-left panel illustrates the area in the source plane (assumed to be at $z=5$ here) that corresponds to the 7' $\times$ '7' square field of view in the image plane (i.e., the sources outside this distorted square will be scattered out of the field of view of the lower-left panel). Note the remarkable power of gravitational lensing in the cluster core, pulling out a number of $z>2$ galaxies from the confusion noise.

a SAFARI spectro-imaging lensing survey of 50 massive clusters $^{1}$. A blank-field survey with the same areal coverage will produce $\sim 8$ detections in the same redshift range, so the lensing survey will offer a factor of $\sim 3$ gain in number. The gain will be even larger if there is an abundance of lower-mass/lower-luminosity galaxies at such high redshift as predicted by hierarchical structure formation theories. Once PAH emitter candidates are found, follow-up spectroscopy can be performed with BLISS to confirm redshifts and examine fainter spectral features. By accessing an epoch at which the space density of IR-luminous galaxies is virtually unknown, SAFARI and BLISS together will

\footnotetext{
1 For this estimate, we made the following assumptions: (1) the infrared luminosity function is in the form derived by Caputi et al. (2007) at $z \sim 2 ;(2) L(I R) \sim 200 \times L(P A H 7.7)$ as observed with $z \sim 2$ SMGs (Pope et al., 2008); (3) a typical cluster has a lensing potential similar to that of A773 (Richard et al., 2009).
}

provide valuable observational data in the epoch of reionization, constraining the cosmic histories of star formation, metallicity evolution (e.g., the $7.7 \mu \mathrm{m}$ PAH strength is sensitive to metallicity), and structure formation.

\section{SPICA's Challenge: Synergy with ALMA/JWST}

In the coming decade, we expect a huge leap with our ability to detect IR-luminous galaxies beyond the current limit of $z \sim 4$. When IR-luminous galaxies are observed in the submm $/ \mathrm{mm}$ range, the strong negative $\mathrm{K}$ correction will compensate for the brightness dimming due to an increased distance, thereby keeping the apparent brightnesses almost constant at $z \sim 1-10$. Although this spectacular effect was anticipated from the very beginning, we have so far not been able to benefit from it mainly for two reasons: (1) Submm/mm observations have not yet achieved a sensitivity with which we can expect a significant number of galaxy detections at $z>4$, and (2) even if we are already seeing a significant number of $z>4$ galaxies in our submm/mm observations, it is often difficult to identify them as such.

ALMA and JWST, together with LMT, CCAT, etc., are likely to change the landscape dramatically. These new observatories will push the frontier of extragalactic IR/submm astronomy beyond a redshift of 4 and probably up to 10 by routinely detecting and identifying dustobscured (i.e., IR-luminous) galaxies at such high redshift.

The main challenge for SPICA is whether it can keep up with these powerful new facilities when probing highredshift Universe. We have discussed here some strategies. Scientifically, the rest-frame mid-IR spectral range, which only SPICA can probe, will be crucial to understand the properties of detected sources (e.g., AGN vs. Star formation though the PAH feature strength). Also, SAFARI will offer significantly faster mapping capabilities with respect to ALMA and JWST, and this may become an important factor if IR-luminous galaxies turn out to be rare at $z>4$.

ACKNOWLEDGEMENTS

I would like to thank Johan Richard for performing the feasibility calculations for the SPICA Lensing Survey.

\section{REFERENCES}

Bouwens, R. J., et al. 2009, ApJ, arXiv:0909.1803

Caputi, D., et al. 2007, ApJ, 660, 97

Clements, D. L., et al. 2007, ApJ, 465, 125

Dunkley, J., et al. 2009, ApJS, 180, 306

Egami, E., et al. 2006, ApJ, 652, L21

Engelbracht, C. W., et al. 2008, ApJ, 678, 804

Fan, X., et al. 2006, AJ, 132, 117

Maiolino, R., et al. 2008, A\&A, 488, 463

Pope, A. et al. 2008, ApJ, 675, 1171

Richard, J. et al. 2009, ApJ, submitted

Smail, I., et al. 2002, MNRAS, 331, 495

Steidel, C. C., et al. 1999, ApJ, 519, 1 